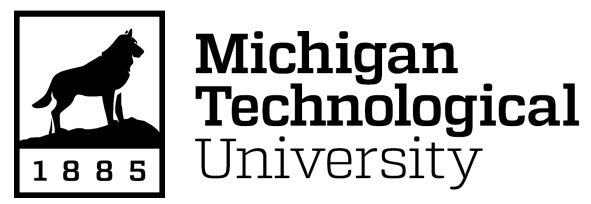

Michigan Technological University Digital Commons @ Michigan Tech

\title{
THE EFFECT OF NUTRITION ON LONGEVITY AND FERTILITY IN DIVERSE DROSOPHILA: A TOR-MEDIATED PROCESS
}

Tessa E. Steenwinkel

Michigan Technological University, testeenw@mtu.edu

Copyright 2021 Tessa E. Steenwinkel

Recommended Citation

Steenwinkel, Tessa E., "THE EFFECT OF NUTRITION ON LONGEVITY AND FERTILITY IN DIVERSE DROSOPHILA: A TOR-MEDIATED PROCESS", Open Access Master's Thesis, Michigan Technological University, 2021.

https://doi.org/10.37099/mtu.dc.etdr/1330

Follow this and additional works at: https://digitalcommons.mtu.edu/etdr

Part of the Genetics Commons, and the Molecular, Genetic, and Biochemical Nutrition Commons 


\title{
THE EFFECT OF NUTRITION ON LONGEVITY AND FERTILITY IN DIVERSE DROSOPHILA: A TOR-MEDIATED PROCESS
}

By

Tessa Elizanne Steenwinkel

\begin{abstract}
A THESIS
Submitted in partial fulfillment of the requirements for the degree of MASTER OF SCIENCE

In Biological Sciences
\end{abstract}

MICHIGAN TECHNOLOGICAL UNIVERSITY

2021

(C) 2021 Tessa E. Steenwinkel 
This thesis has been approved in partial fulfillment of the requirements for the Degree of MASTER OF SCIENCE in Biological Sciences.

\author{
Department of Biological Sciences
}

Thesis Advisor: $\quad$ Dr. Thomas Werner

Committee Member: Dr. Paul Goetsch

Committee Member: Dr. Stephen Techtmann

Department Chair: $\quad$ Dr. Chandrashekhar Joshi 


\section{Table of Contents}

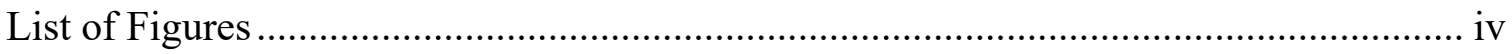

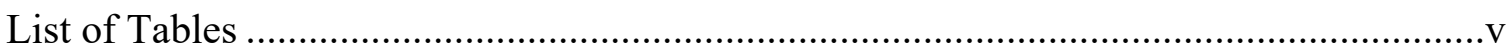

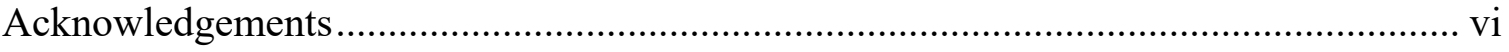

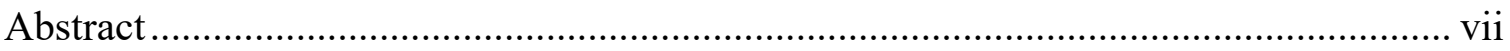

1 Effect of active yeast quantity on diverse Drosophila species .................................1

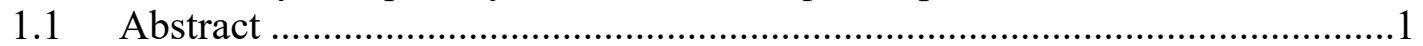

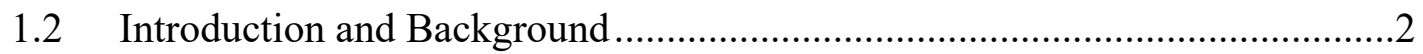

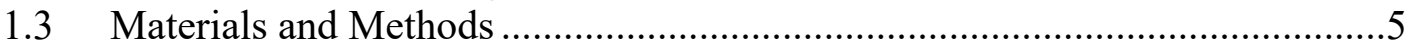

1.3.1 Fecundity and longevity study ………………...............................

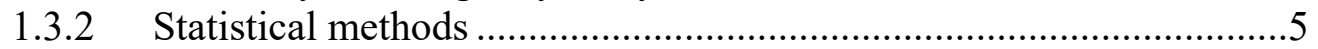

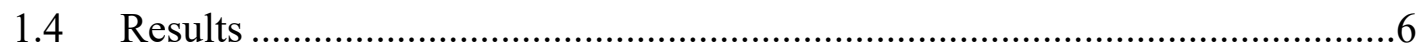

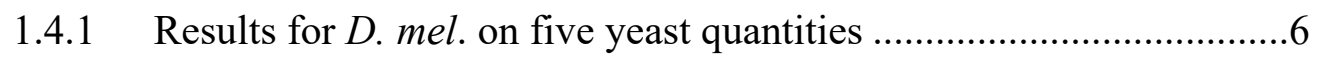

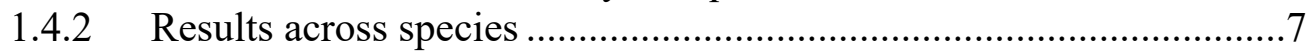

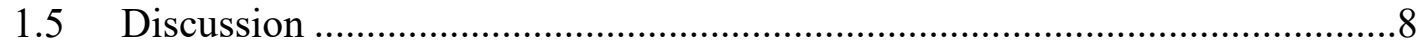

2 Modification of Drosophila metabolism through the use of rapamycin to inhibit the

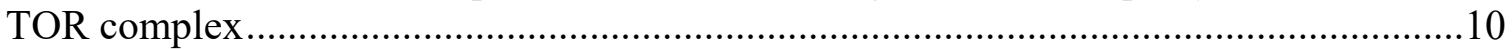

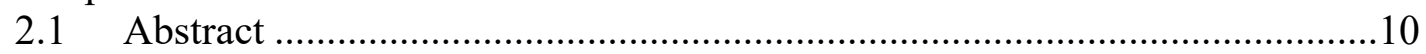

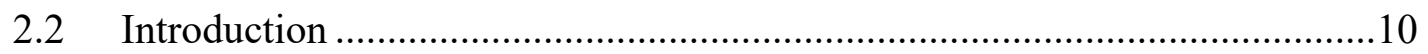

2.3 Materials and Methods ..............................................................................

2.3.1 Use of rapamycin plates in longevity studies ....................................11

2.3.2 Ovariole imaging ....................................................................... 11

2.4 Results ................................................................................................... 11

2.4.1 Results for egg-lay response and longevity with the introduction of rapamycin to an ad libitum diet ...................................................................12

2.4.2 Alterations in lifespan due to the addition of rapamycin to an $\mathrm{ad}$ libitum diet............................................................................................ 12

2.4.3 Results for D. tri. fed with ad libitum amounts of yeast with or without rapamycin ......................................................................................13

2.4.4 Examination of lifespan and fertility of $D$. mel. on a medium diet with and without rapamycin ............................................................................ 14

2.4.5 Images of ovary dissection of D. mel. females fed on various diets.15

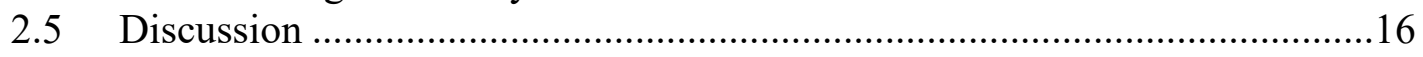

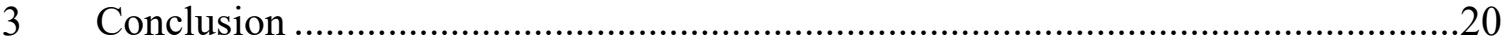

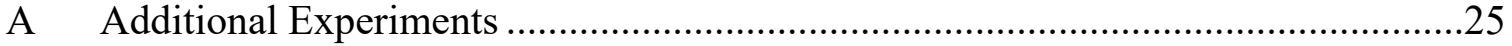

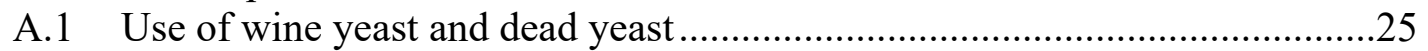

A.2 Western blot for phosphorylated S6K .....................................................25

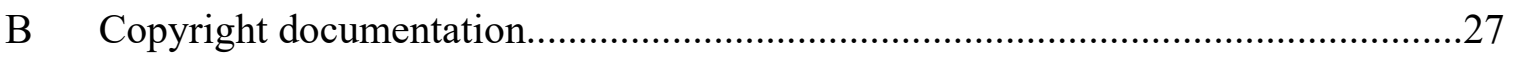

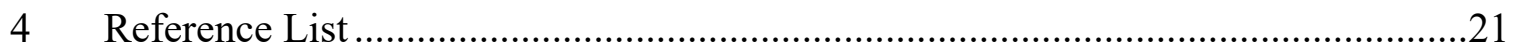




\section{List of Figures}

Figure 1-1 Comparative schemes of the TOR pathway in four different systems.............4

Figure 1-2 Fertility and longevity of $D$. mel. on five dietary treatments .........................6

Figure 1-3 Dietary effect on average egg-lay rates of four Drosophila species .................7

Figure 2-1 Average egg-lay responses of various Drosophila on an ad libitum diet, with

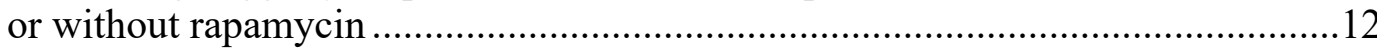

Figure 2-2 Average egg-lay response of $D$. tri. on an ad libitum yeast diet, with or without rapamycin

Figure 2-3 Average egg-lay response of $D$. mel. on a medium diet, with and without

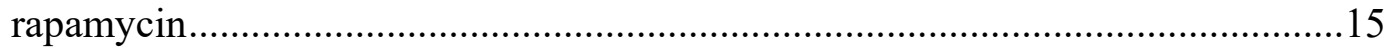

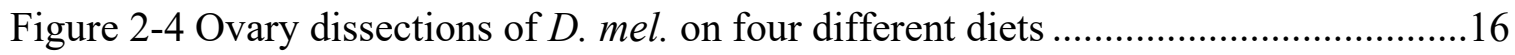

Figure 2-5 Combined graph of the effect of an ad libitum diet on egg-lay rate and both female and male longevity of all four species ................................................ 18

Figure A-1 First attempt at Western blot for detecting S6K phosphorylation...................26

Figure A-2 Second attempt at Western blot for detecting S6K phosphorylation .............26 


\section{List of Tables}

Table 1-1 Median lifespan of female and male Drosophila on three diets........................8

Table 2-1 Median lifespan of various Drosophila on three diets ranging from low to high yeast, where minimal is 1 scoop per day per fly, with or without the addition of

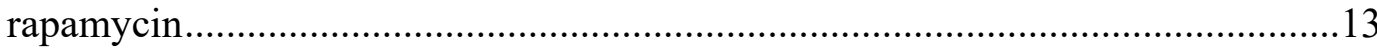

Table 2-2 Median lifespan of $D$. mel. on medium yeast with and without the addition of

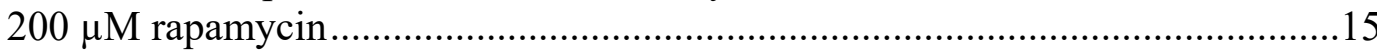




\section{Acknowledgements}

I would like to thank my advisor and mentor, Dr. Thomas Werner, for his endless guidance and support during my undergraduate and now graduate career. I would also like to thank my committee member Dr. Stephen Techtmann for introducing me to R analysis and bioinformatics, as well as Dr. Paul Goetsch for his interest in my project and the possible continuation thereof. I could not have done this without the rest of the Werner Lab, including Kailee Kovach, Mujeeb Shittu, Prajakta Kokate, Will Dion, Lucinda Hall, Morgan Smith, and many others. Also special thanks to Sherry from the Shan lab in assisting us with the Western blots.

This work was made possible by a SURF, a Songer Fellowship, and a Goldwater Fellowship. 


\section{Abstract}

Nutrition and growth are strongly linked, but not much is known about how nutrition leads to growth. To understand the connection between nutrition through diet, growth and/or proliferation, we need to study the phenotypes resulting from the activation and inhibition of central metabolic pathways. Here I use the model organism Drosophila melanogaster (D. mel.) and three non-model Drosophila species with different dietary needs, Drosophila guttifera (D. gut.), Drosophila deflecta (D. def.), and Drosophila tripunctata (D. tri.), to study the effects of dietary amino acid availability in these diverse flies on fecundity and longevity. In addition, I inhibited the Target of Rapamycin (TOR) pathway to test how inhibition interplays with the nutritional stimuli in these four fruit fly species. The hypothesis was that the inhibition of the TOR pathway would reverse the phenotypes observed under conditions of overfeeding. The results show that egg-lay rates increased with higher amino acid availability but decreased in response to TOR inhibition. The longevity data were more varied: most species experienced an increase in median lifespan in both genders with an increase in yeast, except for $D$. mel. females. When exposed to the TOR inhibitor rapamycin, most lifespans decreased, except for $D$. tri. The obtained data will benefit future studies of metabolism and will also help scientists to adopt non-model organisms into their labs to study the true complexity of pathway evolution across different species with diverse nutritional habits and needs. 


\section{Effect of active yeast quantity on diverse Drosophila species}

\subsection{Abstract}

To understand more about the effects of dietary amino acids through the availability of yeast, the model organism Drosophila melanogaster (D. mel.) and three non-model Drosophila species, Drosophila guttifera (D. gut.), Drosophila deflecta $(D$. def.), and Drosophila tripunctata (D. tri.) were exposed to different quantities of yeast in addition to a basic sugar agar. We examined the effect of these different diets on the egglay rate (fecundity) and longevity. Our results show that an increase in yeast leads to an increase in egg production and generally, except for D. mel., an increase in median lifespan. 


\subsection{Introduction and Background}

As with all organisms, Drosophila need nutrition and hydration to survive and function. Currently, nearly 5000 drosophilid species are known ${ }^{1}$, making their diet potentially as diverse as the average human diet. To understand what nutritional components fruit flies need and how the diet affects their ability to live and reproduce, carefully conducted feeding experiments and evaluations of the effects on the life history of fruit flies are necessary. Nutritional science has a long history of studying the relation between nutrition and longevity and/or fecundity in several model organisms; however, much remains unknown. As far as using Drosophila to study nutrition, studies have mainly focused on $D$. mel. and have frequently been inconclusive ${ }^{2}$.

One good reason to study biological phenomena in $D$. mel. is that this important model organism can be quickly and inexpensively cultured in the laboratory and has a well-established genetic toolkit and an annotated genome ${ }^{3}$. Even more importantly, about 70 percent of human disease-causing genes can find homologues in the D. mel. genome ${ }^{4}$, making this fruit fly species an ideal model organism for genetic manipulation and cellular pathway discovery. To truly begin to understand the complexity of the evolution of metabolic pathways and their effects on lifespan and fecundity, we must examine the regulation of these pathways in diverse Drosophila species.

This study utilizes four species of Drosophila, D. mel., D. gut., D. def., and D. tri. These four species are found in the Sophophora subgenus (D. mel.) and the immigranstripunctata radiation (D. gut., D. def., and D. tri.), which have evolved separately over roughly the last 60 million years ${ }^{5}$. Aside from differences in wing and abdominal color patterns, these four species exhibit unique feeding patterns. Although they can all be reared in the lab on standard yeast-sugar food, in nature, they have a wide variety of natural food sources.

$D$. mel. is arguably known as an omnivore ${ }^{6}$ and is most frequently found around decaying fruits and vegetables. The fact that they can eat and reproduce on many fruits and vegetables is greatly advantageous within its heavily crowded ecological niche ${ }^{7}$.

The natural food source of $D$. gut. are mushrooms. Like several other quinaria species group members, when given the choice of mushrooms, bananas, tomatoes, lettuce, or plain agar, they prefer to lay their eggs on mushrooms ${ }^{8}$. D. gut. 's draw towards mushrooms is further supported by their high resistance to mushroom toxins (Werner personal communication), ${ }^{9}$.

D. def., also a quinaria group member, seems to prefer rotting plant matter, especially yellow water lilies ${ }^{10,11}$. Little else is known about $D$. def., making it an interesting organism to study.

Lastly, D. tri. feeds on a mixed diet of decaying fruit and mushrooms, including some that are highly toxic, such as Amanita species ${ }^{9}$. While the mechanisms behind the moderate toxin resistance are unknown, it can be hypothesized that these mechanisms differ from those found in the strongly resistant $D$. gut.

This study focuses on the influence of nutrition on lifespan and fecundity. Lifespan is both a simple and critical tool in the determination of variables in the aging process, both genetically and environmentally ${ }^{12}$. Fecundity in this study is the physiological ability to produce eggs ${ }^{13}$. The first goal of this thesis is to collect baseline data about how different Drosophila species respond to different quantities of yeast. 
The central metabolic pathway that links nutrition, lifespan, and fecundity is known as the TOR (Target of Rapamycin) pathway. The mechanistic TOR (mTOR) pathway, as it is found in mammals, relies on the mTOR protein kinase that is made up of mTORC1 and mTORC2, where mTORC1 responds to nutrients and stress, which then leads to the regulation of homeostasis and growth ${ }^{14}$. mTORC2 is a regulator of cell growth and proliferation, showing effects on autophagy and cancerous cell survival ${ }^{15,16}$. The TOR pathway in Drosophila (dTOR) is highly comparable to mTOR as shown in Figure 1.1. While dTOR lacks some components and some proteins have shifted in the cascade, it provides a valuable opportunity to study TOR in a eukaryotic system without the ethical complications seen in many other model systems and has more similarities to the mTOR system than the more elementary TOR system in Caenorhabditis elegans ${ }^{17-19}$.

The regulation of dTOR has been found to be especially critical in the proliferation and maintenance of germline stem cells in the ovaries of Drosophila, which are crucial to the production of offspring ${ }^{20}$. While flies with Tor null mutations are not viable, hypomorphic Tor mutants show small and distorted ovaries ${ }^{21}$. Interestingly, TOR signaling can be manipulated through diet as shown by Drummond-Barbosa and Spradling (2001). By altering the quantity of protein in the nutrition, they were able to increase the rate of egg production by 60 -fold through the simple addition of nutrients to basic agar ${ }^{22}$. While TOR is not the only player in the proliferation of germline stem cells (ovary follicle maintenance is independent of TOR and involves other interwoven metabolic regulatory pathways), studies on the fecundity of diverse Drosophila species will yield important insights into the importance of TOR complex regulation on reproductive capabilities ${ }^{23}$.

Thus far, we have mainly focused on the effect of TOR on fecundity. However, as mentioned previously, fecundity is only one side of the story. In the discussion of TOR, fecundity must be balanced with lifespan. It is known that dietary, or rather nutritional restriction, increases lifespan ${ }^{2,22,24}$. Evolutionary theory suggests that the lengthening of lifespan in nutritionally restricted conditions is a result of the organism shifting from a focus on reproduction and growth to somatic maintenance ${ }^{25}$. In fruit flies, the shift from proliferation to maintenance can be triggered by limiting the availability of yeast ${ }^{26}$. Kapahi, et al. (2004) further showed that TOR interacts with the insulin-signaling pathway to trigger the maintenance mode through dietary restriction. However, experimentally overexpressing proteins that usually get inhibited by an activated TOR complex has shown to lead to life span extension ${ }^{2,27}$. The overexpression of these downstream TOR targets mimics a nonfunctional TOR complex. The result of an extended lifespan under these mimicked conditions shows that TOR does play a significant role in lifespan in addition to its already established role in fecundity. 


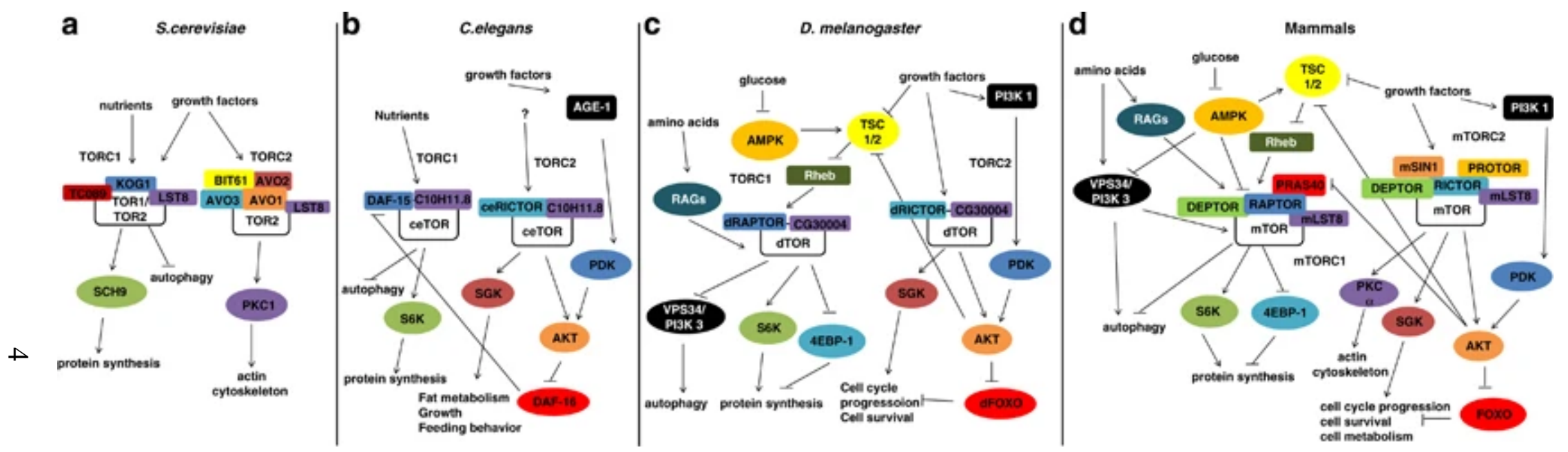

Figure 1-1 Comparative schemes of the TOR pathway in four different systems ${ }^{17}$. Image reproduced from E M Beauchamp et al, with copyright permission from Oncogene (see Appendix B) 
During the course of their lifetime, organisms constantly need to weigh the tradeoff between producing offspring as quickly as possible (possibly at the cost of lifespan) versus reproducing slowly and thereby extending their lifespan to be able to reproduce later during their lifetime ${ }^{28}$. The first part of this study aims to determine, in all four species, if an overabundance of nutrition leads to more egg production in a shorter lifespan, while a restricted diet reduces egg production in favor of prolonging lifespan.

\subsection{Materials and Methods}

\subsubsection{Fecundity and longevity study}

All fly stocks used were maintained at room temperature on food containing brewer's yeast, cornmeal, granulated sugar, agar, and tegosept as an antifungal agent. Canton-S wild-type Drosophila melanogaster were used throughout all experiments. In the second experiment, Drosophila guttifera (15130-1971.10, a kind gift from the Sean B. Carroll lab at UW-Madison), Drosophila deflecta (15130-2018.00, also from the Carroll lab) and Drosophila tripunctata (D.tripunctata_E14\#3, originally wild-caught in Escanaba, MI) were compared against Drosophila melanogaster.

Flies used in the experiments were newly hatched and moved to plain sugar agar medium for overnight acclimatization and to eliminate the weakest flies that die within the first day. On the following morning, ten healthy males and ten females were selected for each experimental sugar agar plate.

Three identical replicates were performed for each experiment and kept concurrently. Each replicate used a negative control, where flies were given no yeast.

On all other plates, scoops of finely ground Baker's yeast powder were measured in 250 nanoliter increments, further referred to as single scoops. Flies were moved to fresh plates daily, using $\mathrm{CO}_{2}$ gas. Fresh plates were prepared a day in advance, refrigerated, and brought to room temperature before transferring the flies. Each vacated plate from the previous 24 hours of incubation with flies was inspected for eggs, and the eggs were counted by arranging them into 10x10 rows and columns. For selected experiments, these eggs were incubated for an additional 24 hours before checking for hatched eggs.

In addition to the numbers of eggs laid and eggs hatched, the number of dead flies and their sex were recorded to measure their lifespan.

\subsubsection{Statistical methods}

All statistics were performed in R Software, primarily using base $\mathrm{R}$ with the stats package. The stats package was used to calculate mean and median values and to perform ANOVA tests with post hoc TukeyHSD. TukeyHSD of multiple comparisons of means with $95 \%$ family-wise confidence levels resulted in significance codes of $p<: 0$; $* * *$,

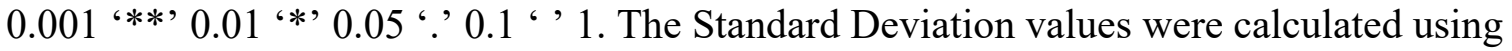
the matrixStats package.

To equalize the measure of egg yields across the species, the egg yields of the first 10 days of reproduction were tracked. Data analysis for the 10-day period were started on the day when all three replicates produced eggs simultaneously. For longevity 
calculations, 30 females and 30 males were monitored in three replicate plates, each holding 10 female and 10 male flies. Food was adjusted daily based on flies remaining from the original 20. Graphs and data analysis for median age combined all three replicates for an overall $n=30$ females and $n=30$ males. Any accidental deaths were disregarded in the conversion to \% survivorship.

\subsection{Results}

In my first experiment, I tested if I can replicate previously published results from other labs, showing that increased nutrition leads to higher egg counts in D. mel., while at the same time decreasing female lifespan. I used the $D$. mel. strain Canton-S to generate baseline data and establish feasibility, after which I extended my research to three additional non-model Drosophila species: D. gut., D. tri., and D. def.

\subsubsection{Results for $D$. mel. on five yeast quantities}

For the first experiment with $D$. mel., I hypothesized that a gradual increase in dietary yeast availability leads to gradually increased fertility. I used five yeast quantities, ranging from the no-yeast control to an ad libitum yeast quantity, where the flies had plenty of food available at all times. My results showed that the hypothesized relationship between nutrition and egg-laying holds true for the female flies of our $D$. mel. strain, i.e., the more yeast they get, the more eggs they lay (Figure 1.2).
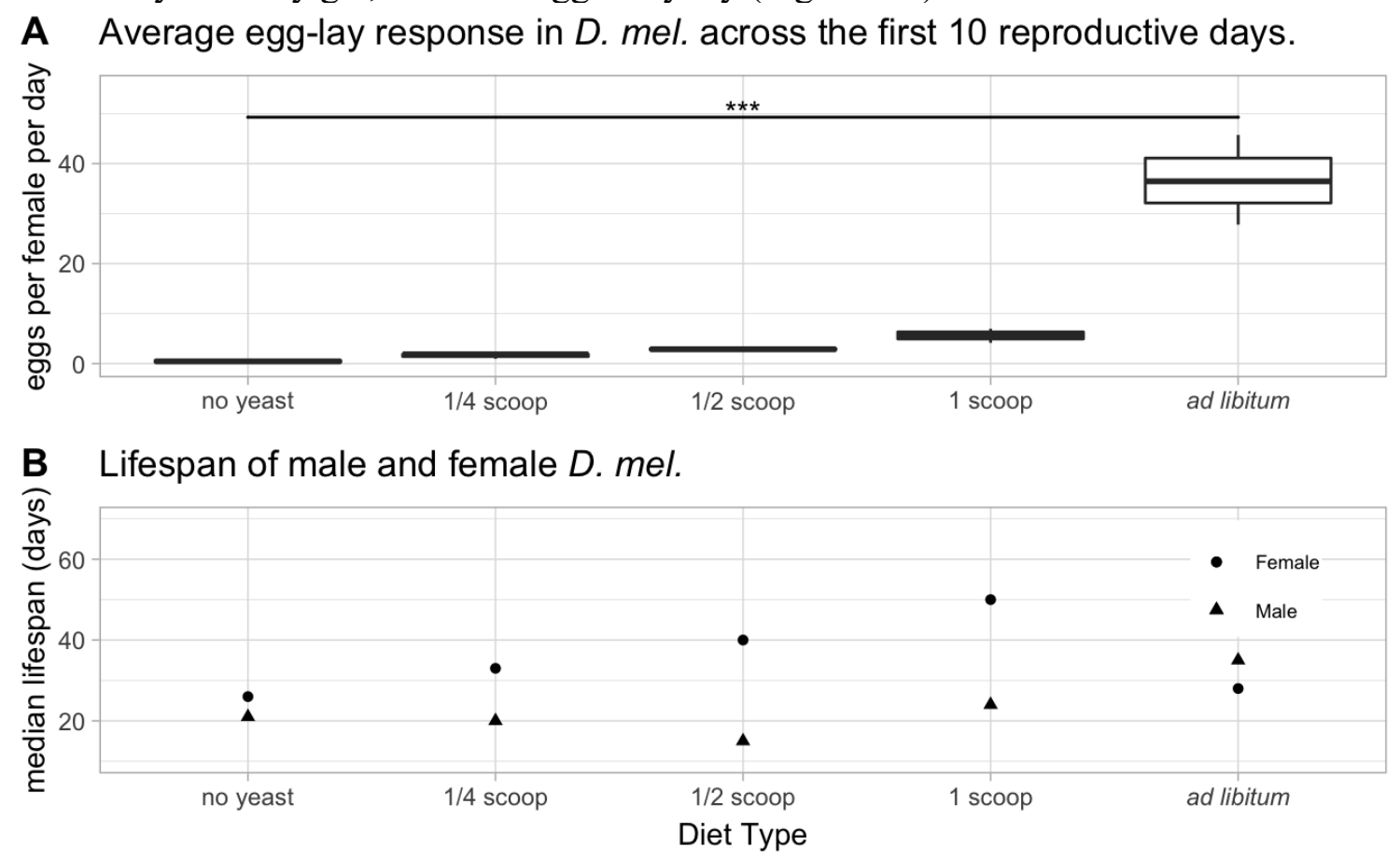


\subsubsection{Results across species}

To further investigate the relationship between nutrition and fecundity, I performed a scaled down investigation (selected yeast quantities) with four species, $D$. mel., D. gut., D. def., and D. tri. As a result, all four species showed similar trends in egg yields, although with different maximum egg-lay capacities (Figure 1.3). Throughout the fecundity investigation, the longevity of both genders was also tracked (Table 1.1). Here, the expected trend of a decrease in female lifespan due to overfeeding was only observed in D. mel. (Table 1.1 and Figure 1.2B), but interestingly not in the three non-model species. Additionally, males of all species experienced extended lifespans with increases in yeast. Particularly, on ad libitum yeast diets, males generally displayed increased lifespans over their female counterparts. However, on lower yeast quantities, males tended to have shorter lifespans than females.

Average egg-lay response across the first 10 reproductive days.
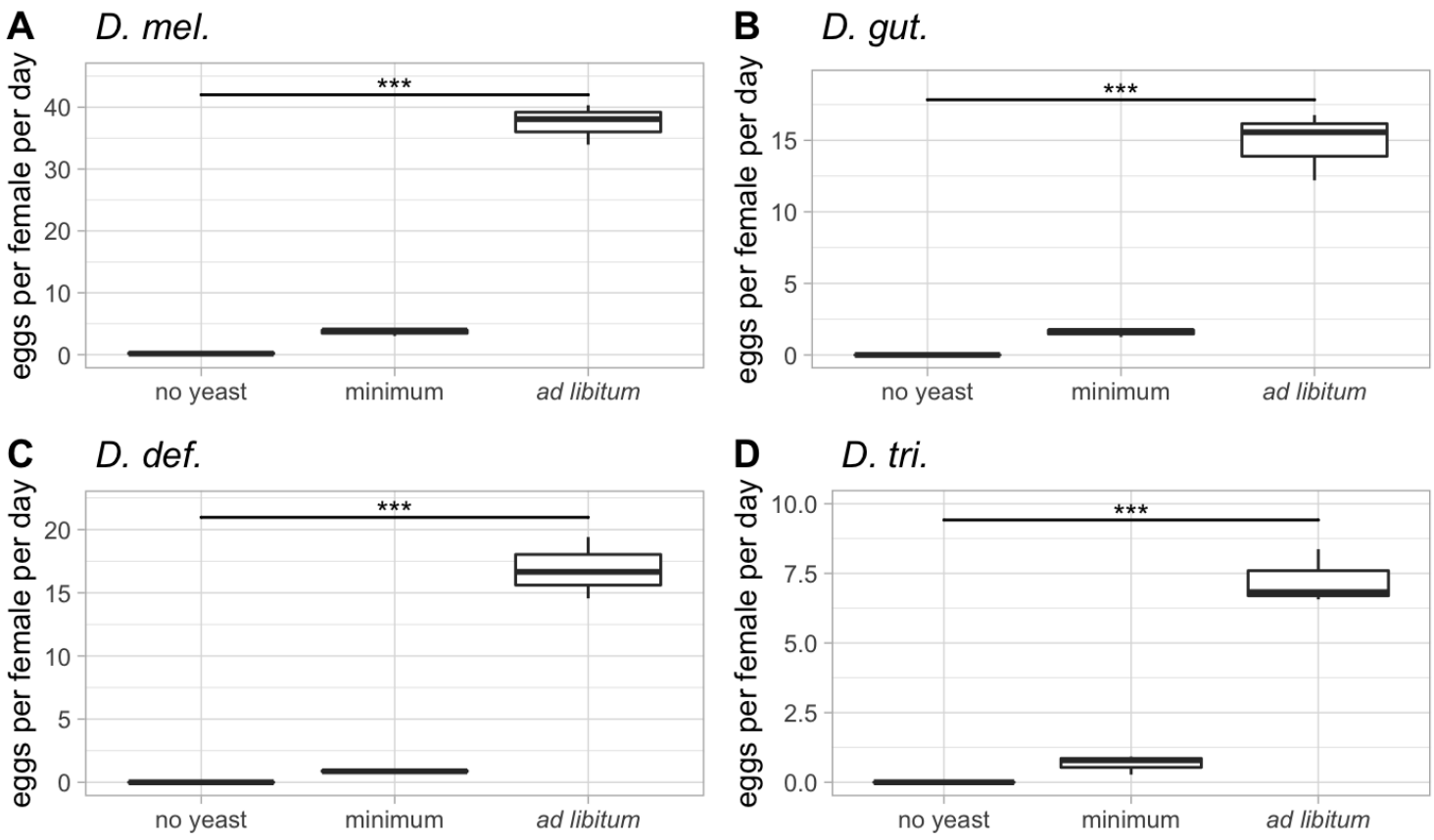
Table 1-1 Median lifespan of female and male Drosophila on three diets. A) Female. B) Male.

\begin{tabular}{lccccccccc}
\multicolumn{3}{l}{ A Median lifespan of female Drosophila. } & \multicolumn{5}{c}{ B Median lifespan of male Drosophila. } \\
\hline \hline species & no yeast & minimum & ad libitum & & species & no yeast & minimum & ad libitum \\
\hline D. mel. & 20 & 29 & 23 & & D. mel. & 17 & 18 & 27 \\
D. gut. & 15 & 26 & 52 & & D. gut. & 13 & 16 & 44 \\
D. def. & 9 & 17 & 35 & & D. def. & 8 & 16 & 74 \\
D. tri. & 14 & 26 & 58 & D. tri. & 12 & 18 & 59 \\
\hline \hline
\end{tabular}

This study started with an in-depth investigation into the effect of dietary amino acids on $D$. mel. fecundity and longevity. The results show that with an increase in yeast, both egg production and lifespan increased, except in females where an abundance of food lowered the median lifespan. This trend in female egg-lay rates and longevity is supported in the Drosophila literature.

The next step was to expand this study into non-model organisms. The study shows that an increase in protein provided through the addition of yeast to a simple sugar agar diet increased the egg production across all four studied Drosophila species, no matter what their natural food sources are, once again supporting and expanding on the trend reported in the Drosophila literature ${ }^{29-31}$.

The topic of lifespan, however, seems to be more complicated when comparing the four species. While $D$. mel. females showed the expected decrease in lifespan in combination with the high amount of yeast, the lifespans of the females of the three other tested species as well as of $D$. mel. males continued to increase when more food was available. This observation is critical to the currently ongoing debate that in reproductively active females, the simple behavior of increasing intake as a response to mating might be shortening their lifespan, as both low and overly high intakes of dietary protein have been shown to shorten lifespan ${ }^{32-36}$. A possible explanation for the decreased lifespan in female $D$. mel. is the observation that $D$. mel. females are the most egg-productive species, increasing their average egg-laying capacity by $\sim 10$-fold, going from 4 eggs per day to almost 40, while the second most egg-productive species, $D$. gut., only saw an increase of 7.5-fold, going from 2 to 15 . The other species, $D$. def. and $D$. tri., have a very low starting point of 1 and 1.5 eggs per day, respectively, and increase to 16 and 6, respectively. Comparatively to $D$. mel., the tested non-model species lay very few eggs. The observed egg production rate differences may mean that more stress is put on a reproducing $D$. mel. female than on females from the other species. The resulting constant proliferation of cells in the $D$. mel. ovaries might contribute to oxidative stress and thus shortening overall lifespan, whereas other species may have more time to recover between births. 
The fact that lifespan is extended in response to increased yeast quantities in our three non-model Drosophila species begs the question if scientists have been relying on the $D$. mel. model system too heavily in the past. What is allowing the other species to thrive on an overabundance of food is an open question. While the other three species see a significant increase in egg-lay rates, the absolute increase is not as dramatic as in $D$. $\mathrm{mel}$. The moderate increase possibly suggests that other species might be more proficient at regulating their metabolic pathway. Better regulation could include storing excess nutrients rather than pouring all energy into proliferation. Tighter regulation on proliferation might be more advantageous when food sources are scarce and the production of offspring is better spread out over the duration of the fly's lifetime, rather than peaking rapidly in the first 10 days. Thus, my discoveries in the three non-model species provide valuable baseline data for future studies that aim to elucidate the pathways that regulate longevity and the regulation of fecundity over a female's lifespan. 


\section{Modification of Drosophila metabolism through the use of rapamycin to inhibit the TOR complex}

\subsection{Abstract}

After examining the effect of the availability of the dietary amino acids on longevity and fecundity in diverse species, we utilized the well-known inhibitor of TOR, rapamycin, to identify the effects of experimental TOR pathway inhibition. We observed major reductions in fecundity (offspring) and, generally, lifespan when the flies were treated with rapamycin. These reductions illustrate the downstream effects of the TOR pathway on metabolism, fecundity, and longevity.

\subsection{Introduction}

The first studies of the TOR pathway were performed 30 years ago ${ }^{37}$. The mTOR protein kinase was discovered through the use of its inhibitor rapamycin. Initially, rapamycin was found to be a potent antifungal agent isolated from Streptomyces hygroscopicus on Easter Island ${ }^{38}$, but identifying its mechanism of action was challenging. When the target of rapamycin was finally identified, it was appropriately called "Target of Rapamycin", TOR ${ }^{39}$. When TOR was shown to be a protein complex, the story got more complicated. It was later shown that TOR can auto-phosphorylate itself ${ }^{40}$, making it evident that the TOR complex acts as a protein kinase ${ }^{37}$.

While one line of research focused on the interaction of rapamycin on the TOR pathway, another focused directly on how rapamycin functions as an antifungal antibiotic. After it was discovered that rapamycin interacts with various metabolic processes, it became evident that rapamycin specifically acts as a suppressor of protein synthesis and growth, thus leading to a halt of cellular proliferation ${ }^{41}$. Rapamycin is essentially able to hold cells in the G1 phase without completely blocking cellular function ${ }^{41}$. Part of this blockage happens through the inhibition of the p70S6 kinase protein, which regulates cell growth and is often upregulated in metastasizing cancers ${ }^{41,42}$.

The study of rapamycin is critical as it is currently used to treat a variety of diseases, from obesity and diabetes to genetic and neurological diseases ${ }^{43}$. Wholebody metabolic effects of rapamycin are difficult to study in larger organisms considering the centrality of this pathway. The centrality also means that any complete knockouts of tor are unviable for study. This, however, leaves the capability to study the effects of dietary rapamycin in diverse Drosophila species.

Previous studies from other labs performed in $D$. mel. discovered that rapamycin caused a decrease in fecundity, while resulting in an increase in lifespan in both gender flies ${ }^{44}$. The increase in lifespan is hypothesized to be one of the effects of starvation, where cells stop producing proteins strictly needed for growth and proliferation, and cells rather focus on proteins needed for maintenance and cellular cleanup ${ }^{45}$. In the presence of rapamycin, one of the mTOR complexes, TORC1, starts 
to send cues to enzymatically break down damaged cellular debris, proteins, lipids, carbohydrates, and other molecules to generate energy ${ }^{45}$.

However, the relationship between rapamycin and longevity is not straightforward, as illustrated by the following studies performed in D. mel. At a concentration of $200 \mu \mathrm{M}$ in the diet, flies experience a bioavailable concentration of rapamycin comparable to studies done in mice ${ }^{24}$. A combination of rapamycin at this concentration and a stringent diet (as far down to $100 \mathrm{~g} / \mathrm{L}$ of yeast in the agar) has shown to extent the longevity of $D$. mel. ${ }^{24,44}$. However, the extended lifespan diminished as food concentrations were increased, while keeping the rapamycin concentration constant ${ }^{44}$. Harrison (2010) found that when the rapamycin concentration is increased between $0-1000 \mu \mathrm{M}$ on a 1:1 yeast : sugar diet with tegosept, the longevity of female flies decreased ${ }^{46}$. Furthermore, when Villa-Cuesta et al. (2014) fed flies on a 12\% yeast diet with rapamycin, ranging from $0-400 \mu \mathrm{M}$, the flies showed no expended lifespan. However, on a $\%$ yeast diet, rapamycin concentrations of $50-200 \mu \mathrm{M}$ rapamycin extended mean longevity, suggesting the possible need for a moderated diet ${ }^{47}$.

The goal of the second chapter of my thesis was to further examine the effect of rapamycin on an ad libitum yeast diet in $D$. mel., as well as the three non-model species D. gut., D. def., and D. tri.

\subsection{Materials and Methods}

\subsubsection{Use of rapamycin plates in longevity studies}

Fly collection and rearing for rapamycin plates was performed as described in 1.3.1. For rapamycin-containing plates, rapamycin powder was added to a final concentration of $200 \mu \mathrm{M}$ by dissolving it in the same ethanol solution used for the incorporation of tegosept.

\subsubsection{Ovariole imaging}

10 male and 10 female flies were reared as described in 1.3.1 on four different diets: 2 scoops of yeast per fly and ad libitum yeast, each on plates with and without rapamycin. The plates were transferred daily to fresh food for 5 consecutive days, after which they were anesthetized with $\mathrm{CO}_{2}$ and drowned in $1 \mathrm{x}$ PBT. The ovaries of the female flies were removed under a dissection microscope in a 9-well plate. The ovaries were then imaged with a dark-field filter at $25 \mathrm{X}$ magnification.

\subsection{Results}

In order to test the effect of rapamycin on the longevity and fecundity of the diverse Drosophila, I tested the flies on $200 \mu \mathrm{M}$ of rapamycin on standard sugar agar plates. Because there was no statistical difference in the egg-lay rates in my previous experiments on low versus no-yeast diets, and because the egg-lay rate was expected 
to decrease further in response to rapamycin, I decided to perform the experiments only on ad libitum quantities of yeast.

\subsubsection{Results for egg-lay response and longevity with the introduction of rapamycin to an ad libitum diet}

As a result of the addition of rapamycin to an ad libitum diet, the females of all four Drosophila species laid significantly fewer eggs (Figure 2.1). D. mel. reduced its egg-lay capacity from 40 eggs per day to only 10 , whereas $D$. gut. decreased from 14 eggs per day to only 4 . D. tri. barely produced any eggs within the first 10 reproductive days, dropping from an average of just over 6 eggs per day to 3. Interestingly, D. def. entirely ceased egg production, which was reminiscent of the minimal diet.

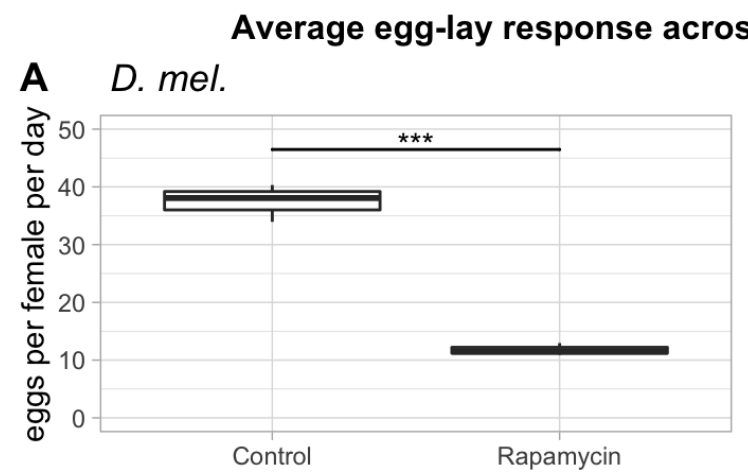

$$
\text { s the first } 10 \text { reproductive days. }
$$
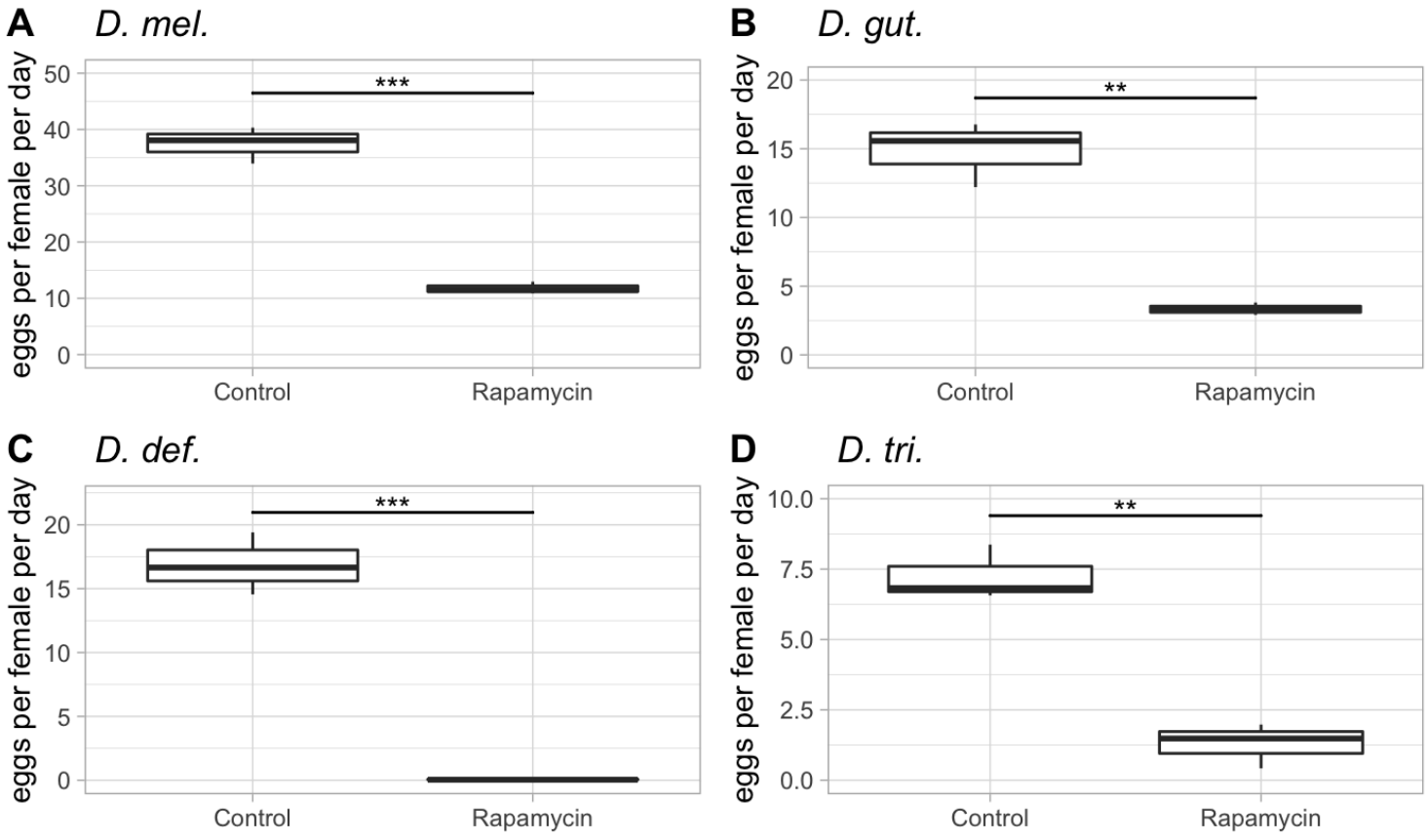

Figure 2-1 Average egg-lay responses of various Drosophila on an ad libitum diet, with or without the addition of rapamycin. A) D. mel. B) D. gut. C) D. def. D) D.tri.

\subsubsection{Alterations in lifespan due to the addition of rapamycin to an ad libitum diet}

I also examined the effect of rapamycin on the median lifespan of the flies on ad libitum yeast quantities. As a result, besides D. tri., all three other species (D. mel., $D$. gut., and $D$. def.), both males and females, showed reduced median lifespans on rapamycin (Table 2.1). 
Table 2-1 Median lifespan of various Drosophila on three diets ranging from low to high yeast, where minimal is 1 scoop per day per fly, with or without the addition of rapamycin. A) female, B) male.

\begin{tabular}{|c|c|c|c|c|c|c|c|c|c|}
\hline \multicolumn{5}{|c|}{ A Median lifespan of female Drosophila. } & \multicolumn{5}{|c|}{ B Median lifespan of male Drosophila. } \\
\hline species & $\begin{array}{l}\text { no } \\
\text { yeast }\end{array}$ & minimum & $\begin{array}{c}\text { ad } \\
\text { libitum }\end{array}$ & $\begin{array}{l}\text { ad libitum } \\
\text { with rapa }\end{array}$ & species & $\begin{array}{c}\text { no } \\
\text { yeast }\end{array}$ & minimum & $\begin{array}{c}\text { ad } \\
\text { libitum }\end{array}$ & $\begin{array}{l}\text { ad libitum } \\
\text { with rapa }\end{array}$ \\
\hline D. mel. & 20 & 29 & 23 & 13 & D. mel. & 17 & 18 & 27 & 16 \\
\hline D. gut. & 15 & 26 & 52 & 18 & D. gut. & 13 & 16 & 44 & 21 \\
\hline D. def. & 9 & 17 & 35 & 28 & D. def. & 8 & 16 & 74 & 37 \\
\hline D. tri. & 14 & 26 & 58 & 74 & D. tri. & 12 & 18 & 59 & 62 \\
\hline
\end{tabular}

\subsubsection{Results for D. tri. fed with ad libitum amounts of yeast with or without rapamycin}

Rapamycin treatment on an ad libitum diet caused a unique response in $D$. tri., as compared to the remaining three tested species: its lifespan unexpectedly increased (Table 2-1). This observation led me to hypothesize a possible resistance mechanism to rapamycin. However, like the other three species, D. tri. showed a reduction in egg-lay rate over the first 10 reproductive days. The first 10 days were chosen because they usually represent the most prolific days in egg production, which is followed by a short plateau and then a decrease of egg-laying until the death of the fly. However, unlike all other species and treatments, the peak of egg-lay production for D. tri. was shifted closer to day 20 in response to rapamycin (Figure 2.3). The peak around day 20, however, is significantly reduced compared to the peak when the flies were not exposed to rapamycin. While a $D$. tri. female fly on an ad libitum diet could lay on average 189 eggs during her lifetime, a female exposed to rapamycin on the same diet would on average only lay 65 eggs. Considering the extended lifespan of $D$. tri. and the later onset of egg production suggests that $D$. tri. adapts to rapamycin during the first week or two of consuming this drug. I speculate that the resistance mechanism(s) in D. tri. may be related to this species' ability to tolerate a variety of mushroom toxins found in its natural diet. Any resistance mechanism, however, would only be partial as egg-laying is significantly reduced. 
Average egg-lay respone of $D$. tri. on varying treatments.

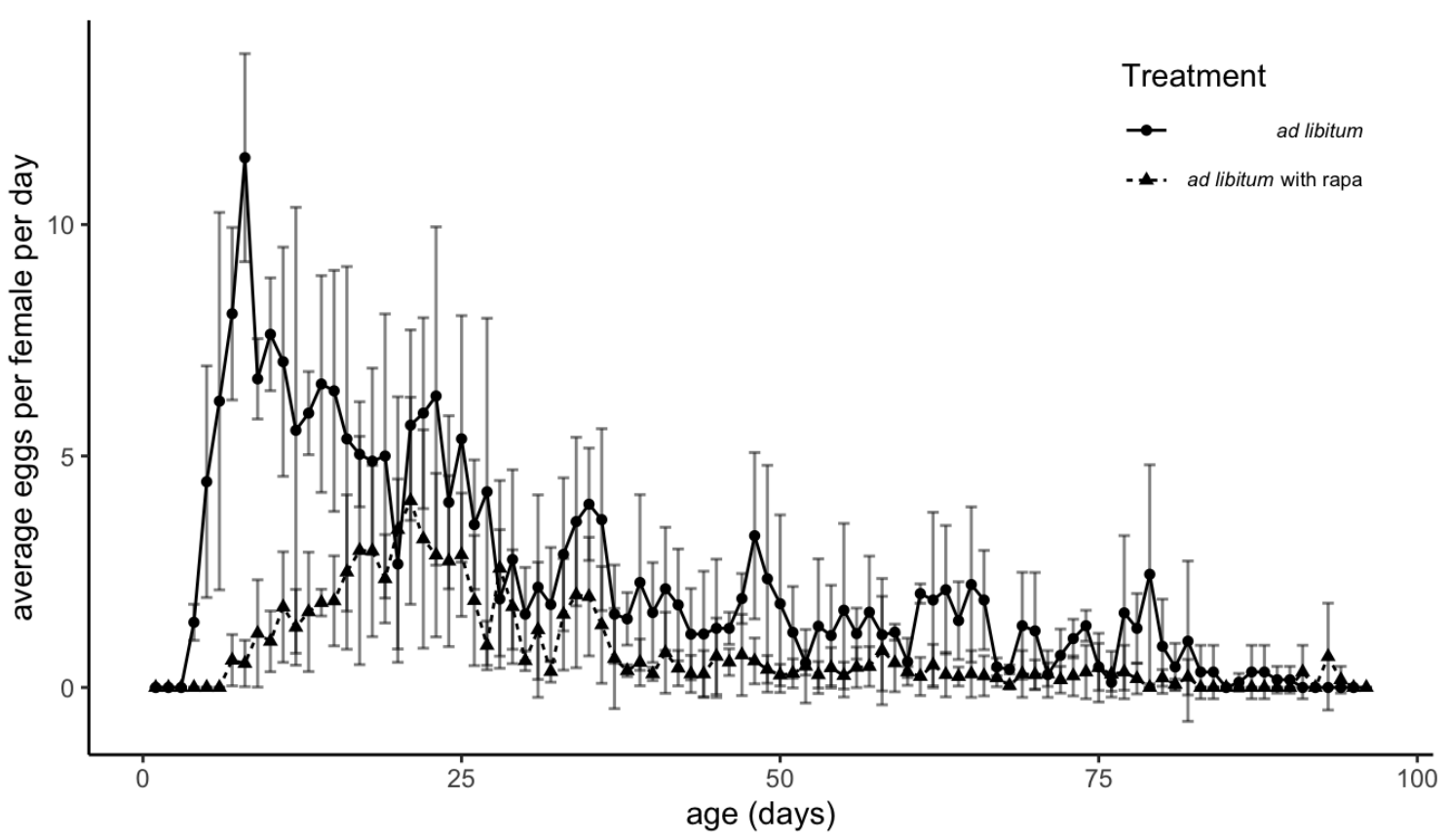

Figure 2-2 Average eggs per female on an ad libitum yeast diet with or without rapamycin. $D$. tri. shows an increased egg production post first 10 reproductive days in response to rapamycin treatment. Error bars showing SD calculated from three replicates each using 10 female flies. Per female adjusted for any deaths during the experiment.

\subsubsection{Examination of lifespan and fertility of $D$. mel. on a medium diet with and without rapamycin}

As I observed in my previous experiment, $D$. mel. females responded to a minimum yeast diet, one scoop, with a longer lifespan than when maintained with a no-yeast or an $a d$ libitum yeast diet. At the same time, the $D$. mel. females were laying a notable number of eggs on the minimum yeast diet. This increased longevity on a restricted diet led me to conduct a more in-depth investigation on the effect of rapamycin in combination with a moderate diet, two scoops per fly. Based on previous studies, I hypothesized that a moderate diet with rapamycin reduces egg-laying but increases their lifespan ${ }^{24,44,46}$. To test if rapamycin can indeed have a life-prolonging effect, I fed $D$. mel. flies with a moderate yeast diet of two scoops in addition to rapamycin. As a result, I observed the expected reduction in the egg-lay rate. However, I did not find evidence of a lifeprolonging effect of the drug. In contrary, rapamycin reduced the lifespan of the flies despite the moderate diet (Figure 2.2 and Table 2.2). These reasons for the reduced longevity are unclear. I noticed black melanin patches on rapamycin-treated flies before they died, indicating an activation of the melanisation host defense pathway, a part of the innate immune response that is normally triggered by parasitoids ${ }^{48}$. I also observed granular crystals in the feces of rapamycin-treated flies, which may be an indicator of 
Average egg-lay response in $D$. mel. across the first 10 reproductive days.

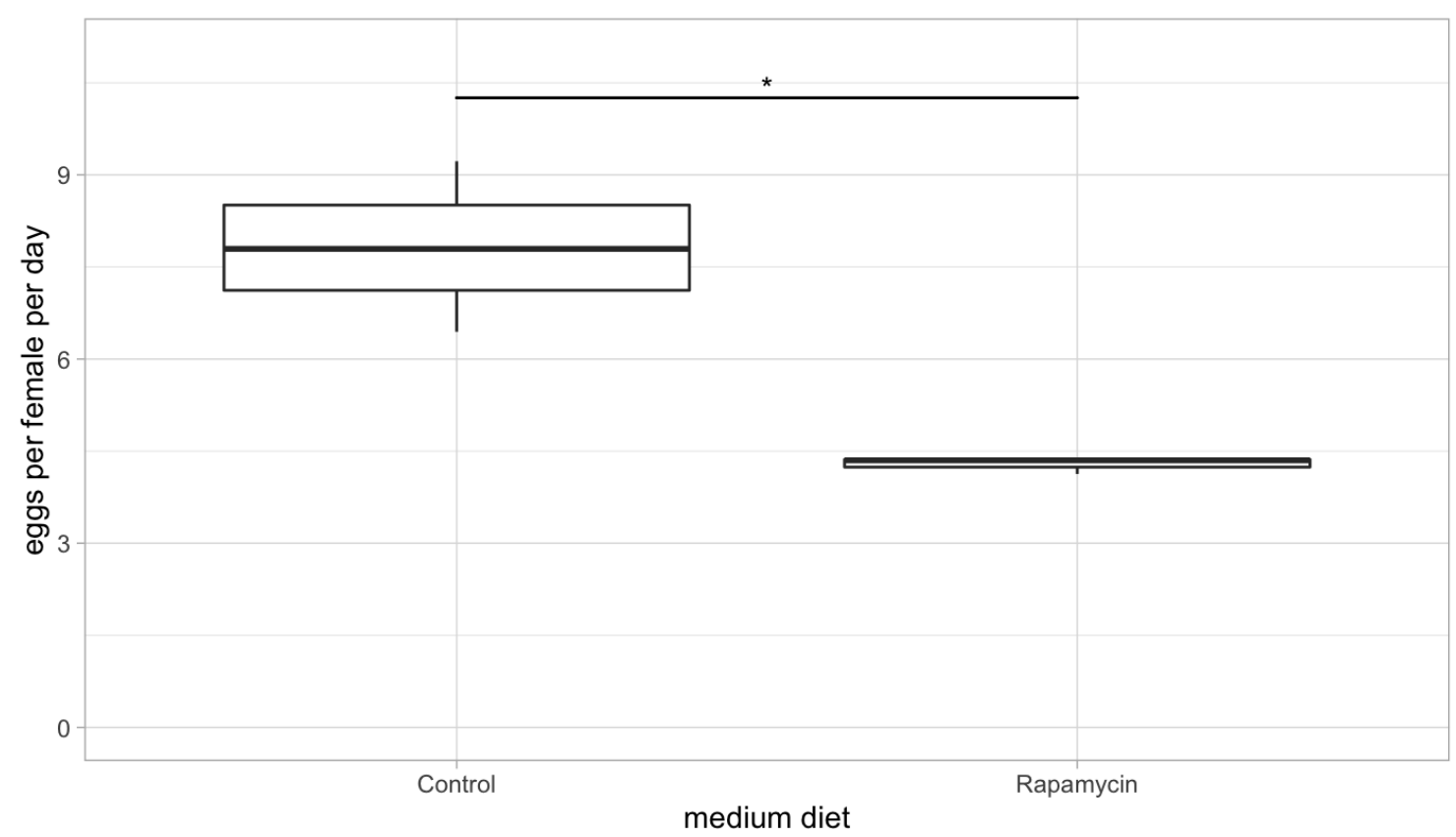

Figure 2-3 Average egg-lay response of $D$. mel. during the first 10 reproductive days on a medium diet with and without rapamycin.

Table 2-2 Median lifespan of $D$. mel. on medium yeast with and without the addition of $200 \mu \mathrm{M}$ rapamycin.

Median Lifespan of $D$. mel. on Two Medium Diets.

\begin{tabular}{lcc}
\hline \hline Sex & Medium Yeast & Medium Yeast with Rapa \\
\hline Females & 24 & 19 \\
Males & 18 & 14 \\
\hline \hline
\end{tabular}

\subsubsection{Images of ovary dissection of $D$. mel. females fed on various diets}

In addition to examining the quantitative responses to nutrition, the ovary dissection gave the opportunity for a qualitative view at the effect of nutrition and rapamycin on the ovaries. $D$. mel. flies of both sexes were put on either the medium diet (two scoops per fly) of yeast or ad libitum with or without rapamycin for 5 days and dissected. Flies fed on the no-rapamycin diets (medium and ad libitum) had welldeveloped, symmetrical, rounded ovaries, while rapamycin-treated flies showed asymmetrical and underdeveloped ovaries (Figure 2-3). 


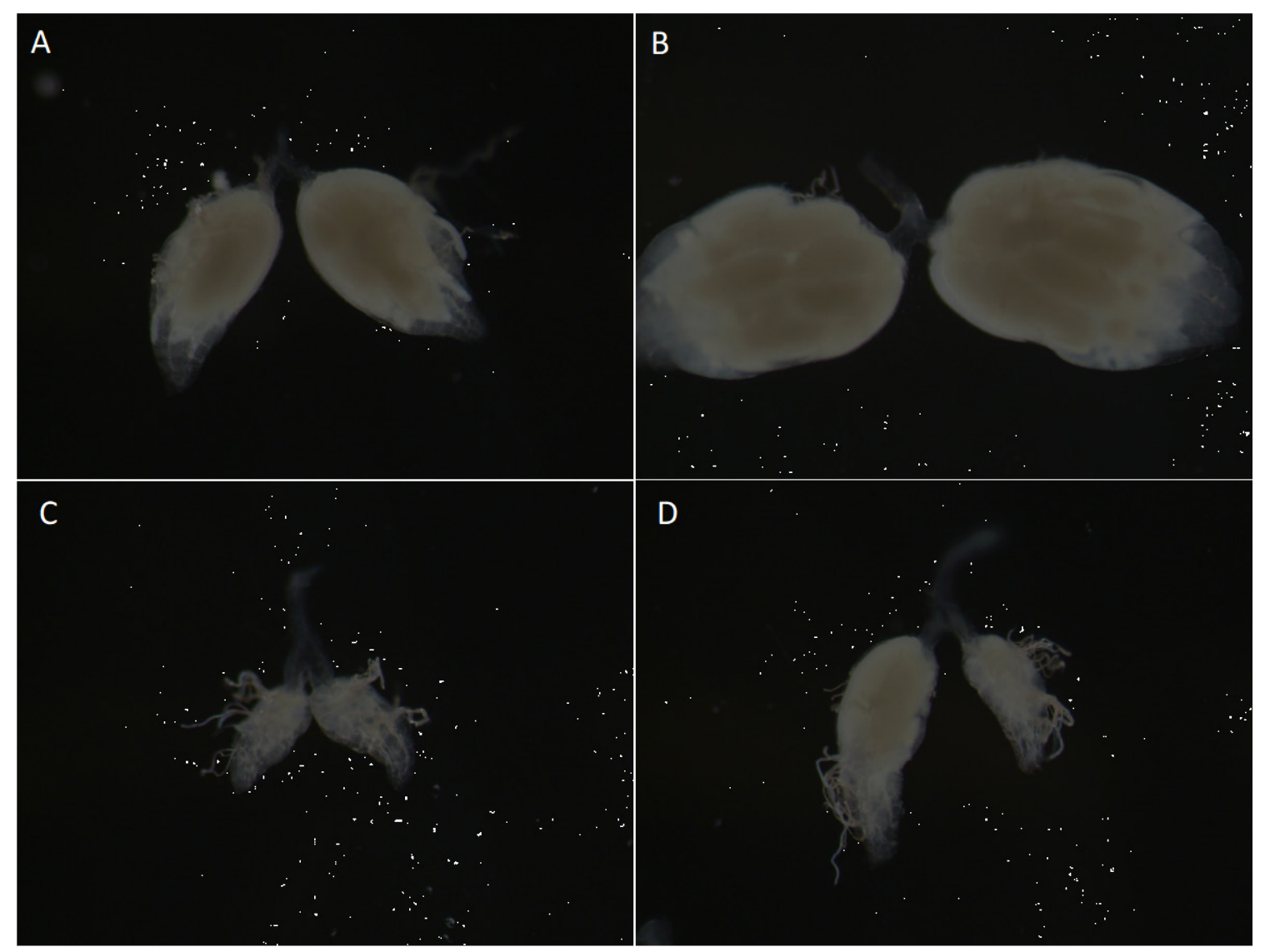

Figure 2-4 Ovary dissections of D. mel. on four different diets. All images taken at 25X magnification. A) D. mel. ovaries on a medium diet (2 scoops/fly). B) ovaries on an $a d$ libitum yeast diet. C) ovaries on medium diet with rapamycin. D) ovaries on an $a d$ libitum yeast diet with rapamycin.

\subsection{Discussion}

As soon as rapamycin is added to an ad libitum yeast diet, the data show a stark trend: egg-laying significantly decreases. The decrease was expected from observations made in other studies using $D$. mel. However, the effects of rapamycin in $D$. mel. have been shown to be genotype-specific ${ }^{49}$. Just like the effects seen under starvation, the inhibition of the TOR protein yields similar egg-lay rates in my experiments for all four species.

Hypothetically rapamycin arrests cell proliferation through the inhibition of the TOR complex, extending lifespan by focusing on cellular maintenance ${ }^{47}$. However, this is not obvious in the data presented here when plenty of nutrition is available. While the dietary amino acids no longer seem to be leading to proliferation, lifespan is not prolonged. If the lack of proliferation indicates cell cycle arrest, then rapamycin must be doing something else to the flies to reduce their lifespans. The one outlier is $D$. tri. with an extended lifespan on rapamycin and a delayed egg-lay peak. I have reasons to believe 
that $D$. tri. might be utilizing a toxin-removal strategy related to its ability to feed on poisonous mushrooms, allowing for the successful adaptation to rapamycin.

Figure 2.5 combines data from parts 1 and 2 .

Average egg-lay response across the first 10 reproductive days and median lifespan.

A D. mel.

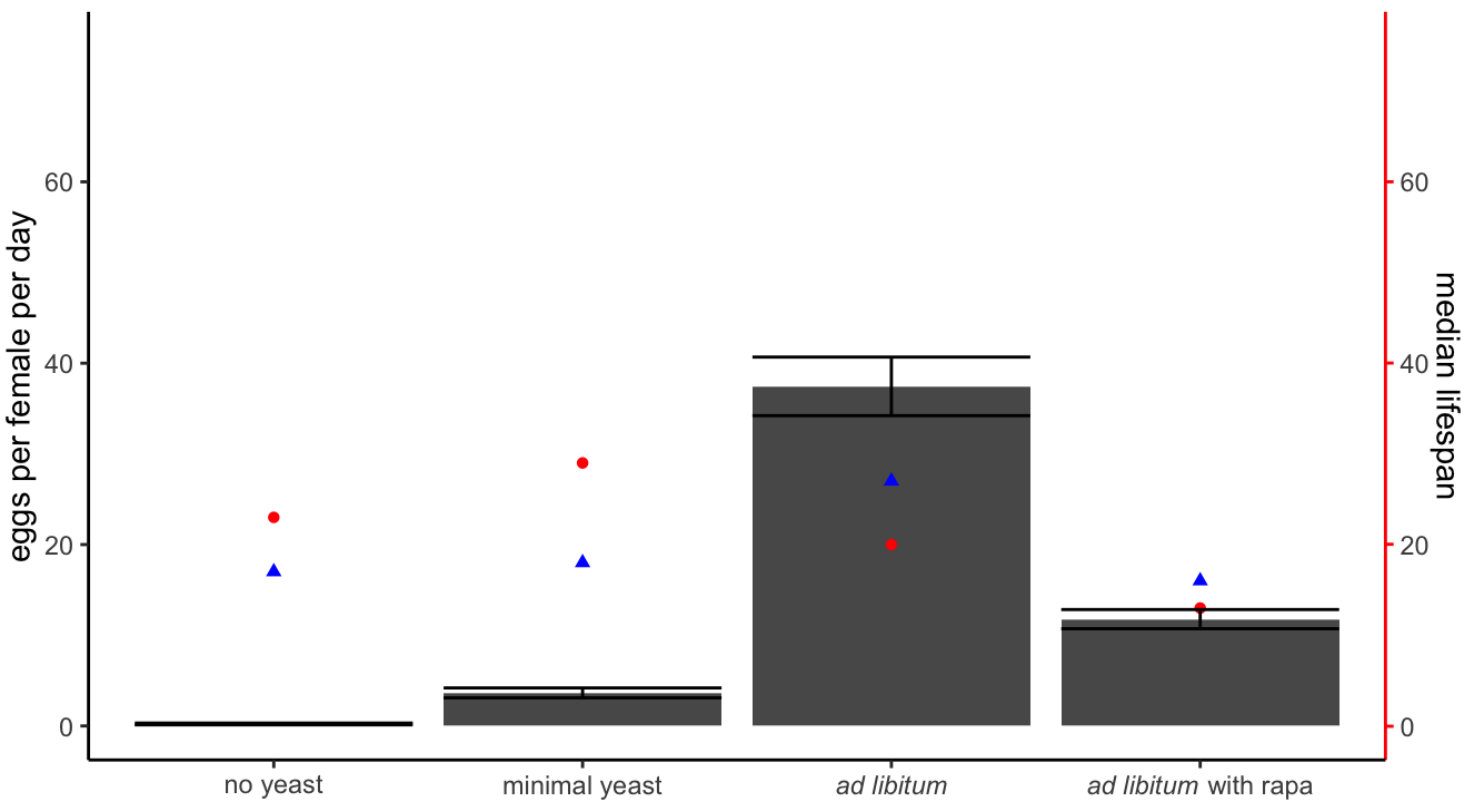

B

D. gut.

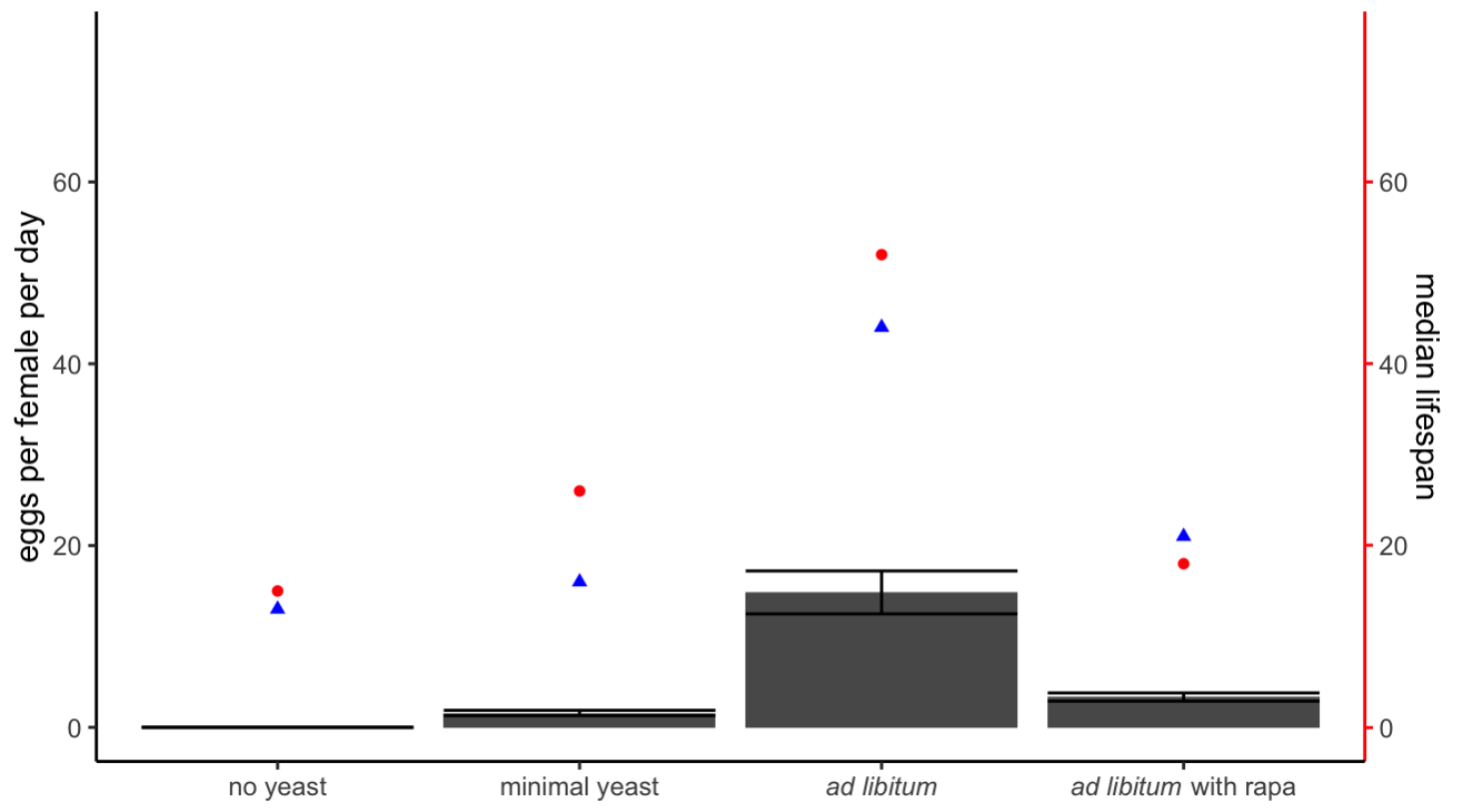


C

D. def.

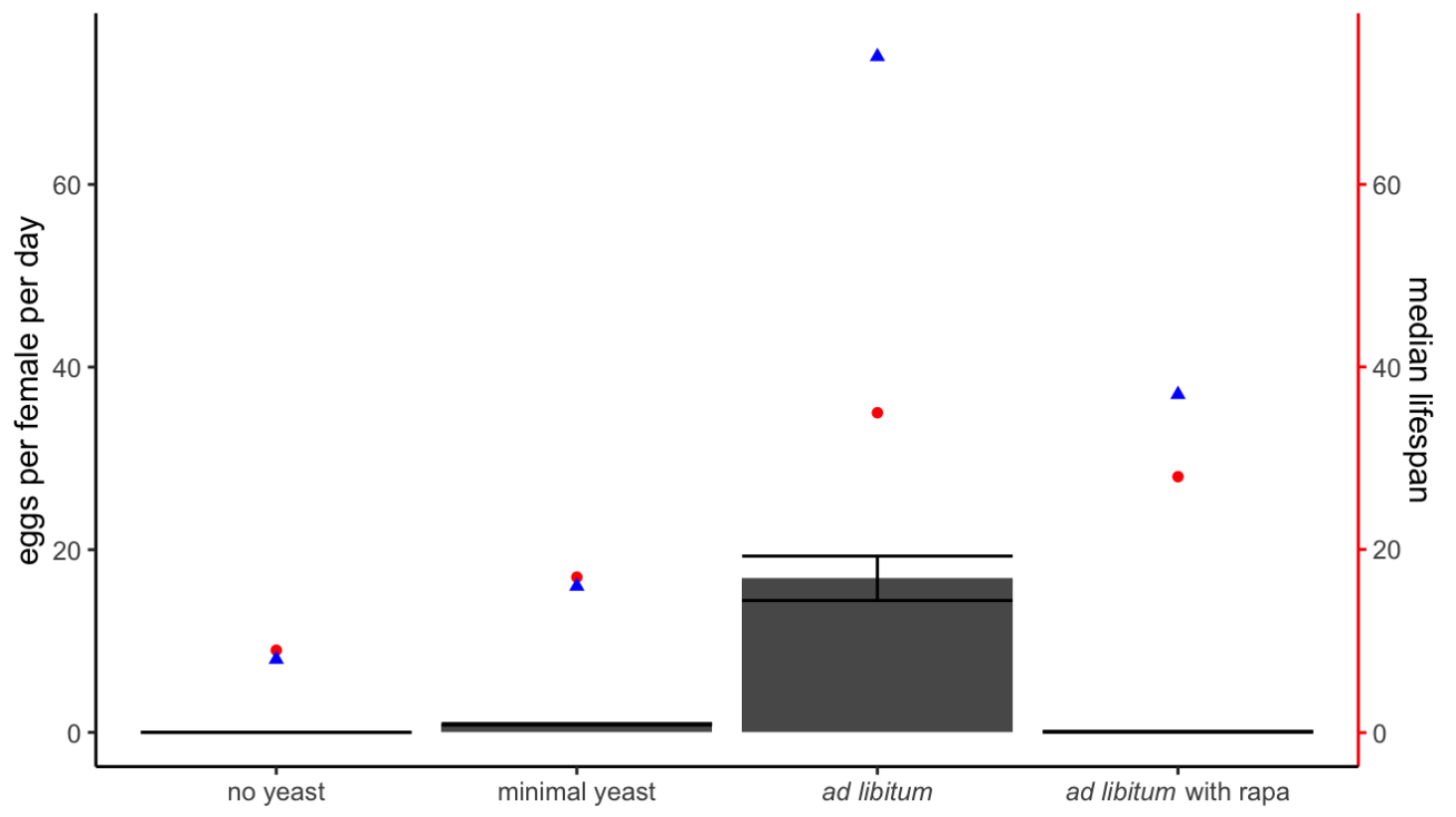

D

D. tri.

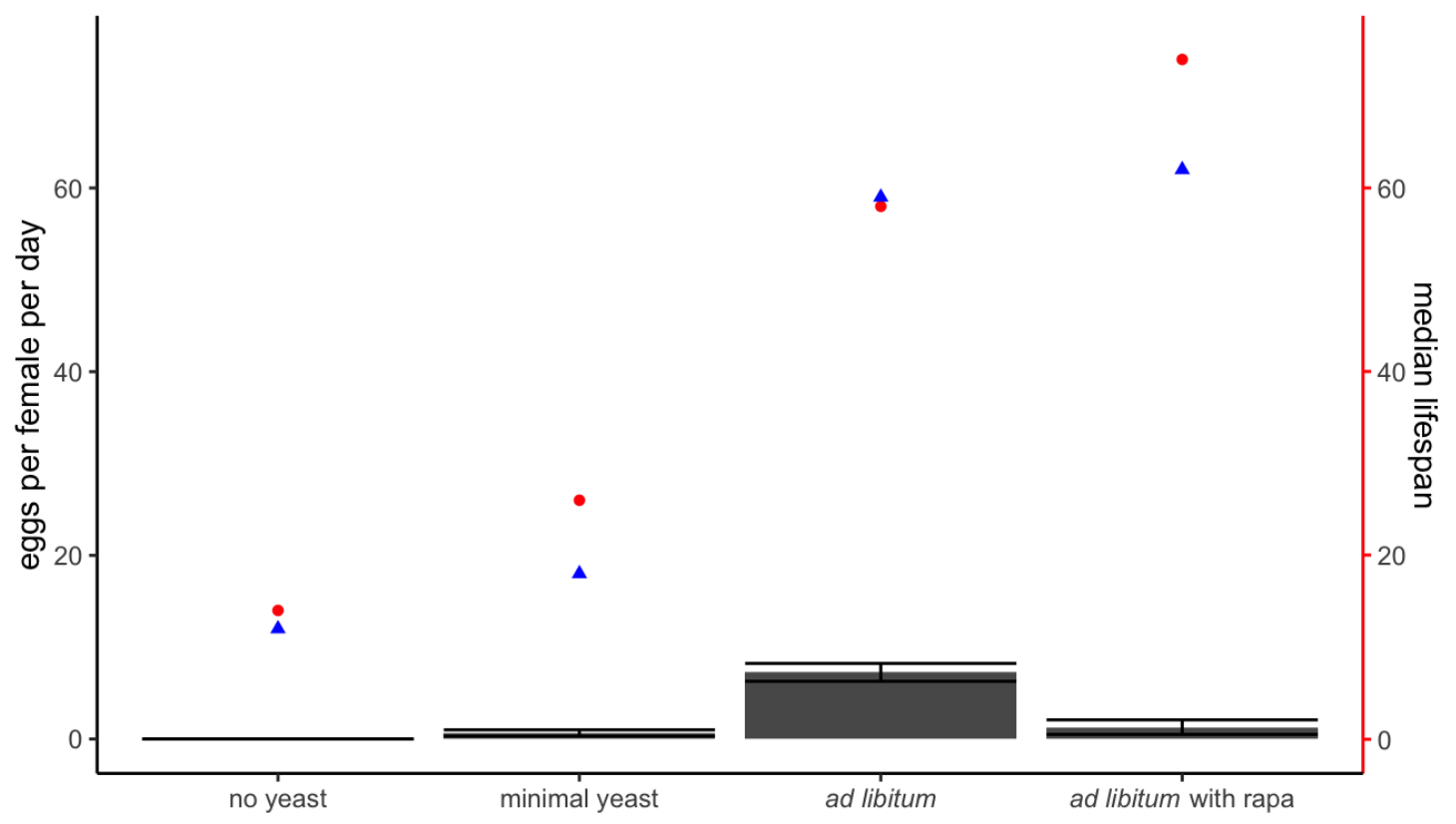

Figure 2-5 Combined graph of the effect of an ad libitum diet on egg-lay rate and both female and male longevity of all four species. Bars represent eggs per female per day, while dots and triangles represent the median lifespan. Red dots are females and blue triangles are males. All scales are equal throughout the figure. 
In summary, it is clear that both overfeeding and the introduction of rapamycin have large influences on the reproductive ability and longevity of diverse Drosophila species. Most notable are the outliers, such as $D$. tri.'s extended lifespan on rapamycin, possibly making it a more robust model organism for the study of longevity, or D. mel. with the significant reduction in lifespan due to overproliferation as a response to overfeeding, making it a good target for studies looking into oxidative stress due to excessive cell replication. $D$. def. is also an interesting species as it stopped any proliferation on nonideal diets, a more extreme phenotype than seen in other species. $D$. def. can also give insight on longevity when females are not producing offspring, in which case they showed a very comparable lifespan between males and females. 


\section{Conclusion}

This thesis examined the effect of nutrition on fecundity and longevity of four fruit fly species: $D$. mel., D. gut., $D$. def., and $D$. tri. I hypothesized that an increase in nutrition activates the metabolic TOR pathway, leading to an increase in proliferation, which would translate into an increased egg production. As a result of this increased egg production, I expected females to suffer a decrease in lifespan. I found that in all studied species, egg-laying in the first 10 days of reproductive activity increased with an increased diet, but only overfed $D$. mel. females experienced a reduction in the median lifespan. After having determined the baseline of the effect of nutrition on fecundity and longevity in four species, the focus of this research shifted towards possible ways to inhibit the activation of the TOR pathway. All four species were exposed to a rapamycin concentration of $200 \mu \mathrm{M}$ in a high-yeast diet, and all species responded with a stark decrease in egg-lay rates in the first 10 reproductive days. Unexpectedly, however, rapamycin did not extend their lifespans, except in $D$. tri. Some of the species-specific results point to the need for more extensive research, but nonetheless underscores the value in using multiple species in future studies.

Overall, my data show the importance of the use of non-model organisms alongside the usual model species and provide insights into nutritional control in the maintenance of diverse fly stocks. This study showed that providing ad libitum access to dietary protein through yeast is not problematic to the flies, while at the same time providing evidence of the well-known claim that the proteins provided by the yeast are essential to proliferation. 


\section{Reference List}

1 Bachli, G. TaxoDros: The database on Taxonomy of Drosophilidae (2021).

2 Mirth, C. K., Nogueira Alves, A. \& Piper, M. D. W. Turning food into eggs: insights from nutritional biology and developmental physiology of Drosophila. Curr Opin Insect Sci 31, 49-57, doi:https://doi.org/10.1016/j.cois.2018.08.006 (2019).

3 Adams, M. D. et al. The genome sequence of Drosophila melanogaster. Science 287, 2185-2195, doi:10.1126/science.287.5461.2185 (2000).

$4 \quad$ Ugur, B., Chen, K. \& Bellen, H. J. Drosophila tools and assays for the study of human diseases. Dis Model Mech 9, 235-244, doi:10.1242/dmm.023762 (2016).

5 O'Grady, P. M. \& DeSalle, R. Phylogeny of the Genus Drosophila. Genetics 209, 1-25, doi:10.1534/genetics.117.300583 (2018).

6 Yang, D. Carnivory in the larvae of Drosophila melanogaster and other Drosophila species. Sci Rep 8, 15484, doi:10.1038/s41598-018-33906-w (2018).

$7 \quad$ Keller, A. Drosophila melanogaster's history as a human commensal. Curr Biol 17, R77-R81, doi:10.1016/j.cub.2006.12.031 (2007).

8 Bunyard, B. \& Foote, B. A. Biological notes on Drosophila guttifera (Diptera: Drosophilidae), a consumer of mushrooms. Entomol news 101 (1990).

9 Scott Chialvo, C. H. \& Werner, T. Drosophila, destroying angels, and deathcaps! Oh my! A review of mycotoxin tolerance in the genus Drosophila. Front biol 13, 91-102, doi:10.1007/s11515-018-1487-1 (2018).

10 Werner, T., Steenwinkel, T. \& Jaenike, J. The Encyclopedia of North American Drosophilids Volume 1: Drosophilids of the Midwest and Northeast. 3 edn, Vol. 1 (Michigan Technological University, 2018).

11 Werner, T., Steenwinkel, T. \& Jaenike, J. The Encyclopedia of North American Drosophilids Volume 2: Drosophilids of the Southeast. Vol. 2 (Michigan Technological University 2020).

12 Sun, Y. et al. Aging studies in Drosophila melanogaster. Methods Mol Biol 1048, 77-93, doi:10.1007/978-1-62703-556-9_7 (2013).

13 Novoseltsev, V. N., Novoseltseva, J. A. \& Yashin, A. I. What does a fly's individual fecundity pattern look like? The dynamics of resource allocation in reproduction and ageing. Mech Ageing Dev 124, 605-617, doi:10.1016/s00476374(03)00061-7 (2003).

14 McCormick, M. A., Tsai, S.-Y. \& Kennedy, B. K. TOR and ageing: a complex pathway for a complex process. Philos Trans R Soc Lond B Biol Sci 366, 17-27, doi:10.1098/rstb.2010.0198 (2011).

15 Kuo, Y., Huang, H., Cai, T. \& Wang, T. Target of Rapamycin Complex 2 regulates cell growth via Myc in Drosophila. Sci Rep 5, 10339, doi:10.1038/srep10339 (2015).

16 Schaub, T. et al. mTORC1 and mTORC2 Differentially Regulate Cell Fate Programs to Coordinate Osteoblastic Differentiation in Mesenchymal Stromal Cells. Sci Rep 9, 20071, doi:10.1038/s41598-019-56237-w (2019).

17 Beauchamp, E. M. \& Platanias, L. C. The evolution of the TOR pathway and its role in cancer. Oncogene 32, 3923-3932, doi:10.1038/onc.2012.567 (2013). 
18 Zhang, H., Stallock, J. P., Ng, J. C., Reinhard, C. \& Neufeld, T. P. Regulation of cellular growth by the Drosophila target of rapamycin dTOR. Genes Dev 14, 2712-2724, doi:10.1101/gad.835000 (2000).

19 Blackwell, T. K., Sewell, A. K., Wu, Z. \& Han, M. TOR Signaling in Caenorhabditis elegans Development, Metabolism, and Aging. Genetics 213, 329360, doi:10.1534/genetics.119.302504 (2019).

20 Lafever, L., Feoktistov, A., Hsu, H.-J. \& Drummond-Barbosa, D. Specific roles of Target of rapamycin in the control of stem cells and their progeny in the Drosophila ovary. Development 137, 2117-2126, doi:10.1242/dev.050351 (2010).

21 Zhang, Y., Billington, C. J., Pan, D. \& Neufeld, T. P. Drosophila target of rapamycin kinase functions as a multimer. Genetics 172, 355-362, doi:10.1534/genetics.105.051979 (2006).

22 Drummond-Barbosa, D. \& Spradling, A. C. Stem cells and their progeny respond to nutritional changes during Drosophila oogenesis. Dev Biol 231, 265-278, doi:10.1006/dbio.2000.0135 (2001).

23 Armstrong, A. R., Laws, K. M. \& Drummond-Barbosa, D. Adipocyte amino acid sensing controls adult germline stem cell number via the amino acid response pathway and independently of Target of Rapamycin signaling in Drosophila. Development 141, 4479-4488, doi:10.1242/dev.116467 (2014).

24 Bass, T. M. et al. Optimization of dietary restriction protocols in Drosophila. $J$ Gerontol A Biol Sci Med Sci 62, 1071-1081, doi:10.1093/gerona/62.10.1071 (2007).

25 Holliday, R. Food, reproduction and longevity: is the extended lifespan of calorierestricted animals an evolutionary adaptation? Bioessays 10, 125-127, doi:10.1002/bies.950100408 (1989).

26 Mair, W., Piper, M. D. W. \& Partridge, L. Calories Do Not Explain Extension of Life Span by Dietary Restriction in Drosophila. PLoS Biol 3, e223, doi:10.1371/journal.pbio.0030223 (2005).

27 Kapahi, P. et al. Regulation of lifespan in Drosophila by modulation of genes in the TOR signaling pathway. Curr Biol 14, 885-890, doi:10.1016/j.cub.2004.03.059 (2004).

28 Rogina, B. et al. Distinct biological epochs in the reproductive life of female Drosophila melanogaster. Mech Ageing Dev 128, 477-485, doi:10.1016/j.mad.2007.06.004 (2007).

29 Piper, M. D. et al. A holidic medium for Drosophila melanogaster. Nat Methods 11, 100-105, doi:10.1038/nmeth.2731 (2014).

30 Bradley, T. J. \& Simmons, F. H. An analysis of resource allocation in response to dietary yeast in Drosophila melanogaster. J Insect Physiol 43, 779-788, doi:10.1016/s0022-1910(97)00037-1 (1997).

31 Terashima, J. \& Bownes, M. Translating available food into the number of eggs laid by Drosophila melanogaster. Genetics 167, 1711-1719, doi:10.1534/genetics.103.024323 (2004). 
32 Chapman, T. \& Partridge, L. Female fitness in Drosophila melanogaster: an interaction between the effect of nutrition and of encounter rate with males. Proc Royal Soc B 263, 755-759, doi:10.1098/rspb.1996.0113 (1996).

33 Mair, W., Piper, M. D. \& Partridge, L. Calories do not explain extension of life span by dietary restriction in Drosophila. PLoS Biol 3, e223, doi:10.1371/journal.pbio.0030223 (2005).

34 Partridge, L. \& Gems, D. Mechanisms of aging: public or private? Nat Rev Genes 3, 165-175, doi:10.1038/nrg753 (2002).

35 Partridge, L., Piper, M. D. \& Mair, W. Dietary restriction in Drosophila. Mech Ageing Dev 126, 938-950, doi:10.1016/j.mad.2005.03.023 (2005).

36 Piper, M. D. \& Partridge, L. Dietary restriction in Drosophila: delayed aging or experimental artefact? PLoS Genet 3, e57, doi:10.1371/journal.pgen.0030057 (2007).

37 Sabatini, D. M. Twenty-five years of mTOR: Uncovering the link from nutrients to growth. Proc. Natl. Acad. Sci. U.S.A. 114, 11818-11825, doi:10.1073/pnas.1716173114 (2017).

38 Vézina, C., Kudelski, A. \& Sehgal, S. N. Rapamycin (AY-22,989), a new antifungal antibiotic. I. Taxonomy of the producing streptomycete and isolation of the active principle. J Antibiot (Tokyo) 28, 721-726, doi:10.7164/antibiotics.28.721 (1975).

39 Sabers, C. J. et al. Isolation of a Protein Target of the FKBP12-Rapamycin Complex in Mammalian Cells. J Biol Chem 270, 815-822, doi:10.1074/jbc.270.2.815 (1995).

40 Brown, E. J. et al. Control of p70 s6 kinase by kinase activity of FRAP in vivo. Nature 377, 441-446, doi:10.1038/377441a0 (1995).

41 Terada, N., Takase, K., Papst, P., Nairn, A. C. \& Gelfand, E. W. Rapamycin inhibits ribosomal protein synthesis and induces G1 prolongation in mitogenactivated T lymphocytes. J Immunol 155, 3418-3426 (1995).

42 Harada, H., Andersen, J. S., Mann, M., Terada, N. \& Korsmeyer, S. J. p70S6 kinase signals cell survival as well as growth, inactivating the pro-apoptotic molecule BAD. Proc Natl Acad Sci U S A 98, 9666-9670, doi:10.1073/pnas.171301998 (2001).

43 Li, J., Kim, S. G. \& Blenis, J. Rapamycin: one drug, many effects. Cell Metab 19, 373-379, doi:10.1016/j.cmet.2014.01.001 (2014).

44 Bjedov, I. et al. Mechanisms of life span extension by rapamycin in the fruit fly Drosophila melanogaster. Cell Metab 11, 35-46, doi:10.1016/j.cmet.2009.11.010 (2010).

45 Lum, J. J., DeBerardinis, R. J. \& Thompson, C. B. Autophagy in metazoans: cell survival in the land of plenty. Nat Rev Mol Cell Biol 6, 439-448, doi:10.1038/nrm1660 (2005).

46 Harrison, B., Tran, T. T., Taylor, D., Lee, S. D. \& Min, K. J. Effect of rapamycin on lifespan in Drosophila. Geriatr Gerontol Int 10, 110-112, doi:10.1111/j.14470594.2009.00569.x (2010). 
47 Villa-Cuesta, E., Fan, F. \& Rand, D. M. Rapamycin reduces Drosophila longevity under low nutrition. IOSR J Pharm 4, 43-51, doi:10.9790/3013-0408043051 (2014).

48 Dudzic, J. P., Hanson, M. A., Iatsenko, I., Kondo, S. \& Lemaitre, B. More Than Black or White: Melanization and Toll Share Regulatory Serine Proteases in Drosophila. Cell Rep 27, 1050-1061.e1053, doi:10.1016/j.celrep.2019.03.101 (2019).

49 Rohde, P. D. et al. Genotype and Trait Specific Responses to Rapamycin Intake in Drosophila melanogaster. Insects 12, 474, doi:10.3390/insects 12050474 (2021). 


\section{A Additional Experiments}

In addition to the experiments described in the main body of this thesis, further experiments were performed that could be the starting point to new projects or branches of this research.

\section{A.1 Use of wine yeast and dead yeast}

While the experiments shown in the main part of this thesis were performed using instant live Baker's yeast, I also performed experiments with all four Drosophila species on wine yeast and heat-killed Baker's. I used three levels of diets: no yeast, one scoop of yeast, and ad libitum yeast.

The reason for testing wine yeast was to explore what yeast type would be best for our experiments. It was hypothesized that wine yeast would work well but not as well as Baker's yeast, as D. mel. is commonly reared in the lab on Baker's yeast. This hypothesis proved to be true. Both ad libitum diets of Baker's yeast and wine yeast resulted in a statistical increase in egg-lay rate over the 1 scoop egg-lay rate; however, lower statistical significance was observed with the use of wine yeast. Thus, in order increase the likeliness of obtaining significant results for the main experiments between different yeast levels, only Baker's yeast was used.

The idea of killing Baker's yeast by baking it at $400^{\circ} \mathrm{F}$ for 30 minutes had similar expectations to that of using undesirable types of yeast. However, while dead yeast should contain the same nutrients, the flies were not interested in eating it. The yeast remained uneaten, and results in egg-lay rate and lifespan did not differ from those on control plates with no yeast. This result led to the exclusion of this path of research from the work in this thesis. However, further investigations could be performed to test how adding ethanol to the dead yeast will affect the flies' appetite to highlight the importance of smell in the consumption of a diet.

\section{A.2 Western blot for phosphorylated S6K}

Most of this thesis focuses on obtaining a quantitative measure of the effect of nutrition on longevity and fertility. It is hypothesized in the literature that longevity and fertility are regulated by the TOR pathway. However, longevity and fertility are far downstream effects of TOR and influenced by other molecular pathways and conditions. In order to provide additional evidence that the alterations of longevity and fertility are due to changes in TOR signaling, I utilized the well-known inhibitor of rapamycin to reverse the phenotypes seen in overfed flies. Hypothetically, providing an ad libitum diet will overactivate the TOR complex. This overactivation, in turn, should be reduced in the presence of the TOR complex inhibitor rapamycin. As a direct effect of the rapamycin treatment, there should be a reduction in phosphorylated S6K, a downstream target of the central kinase in the TOR pathway, as shown by Bjedov et al. (2010). As Bjedov et al. (2010) showed, the reduction in phosophorylated S6K should be observable through a Western blot. However, two separate attempts at performing Western blots, with the 
gracious help of Xinqian (Sherry) Chen form the Shan lab, did not show phosphorylated $\mathrm{S} 6 \mathrm{~K}$ in control flies or rapamycin-treated flies. Trouble shooting and identifying alternative ways of protein isolation from flies could further molecular research into the TOR pathway. However, due to time limitations, follow-up experiments were not feasible for this thesis.

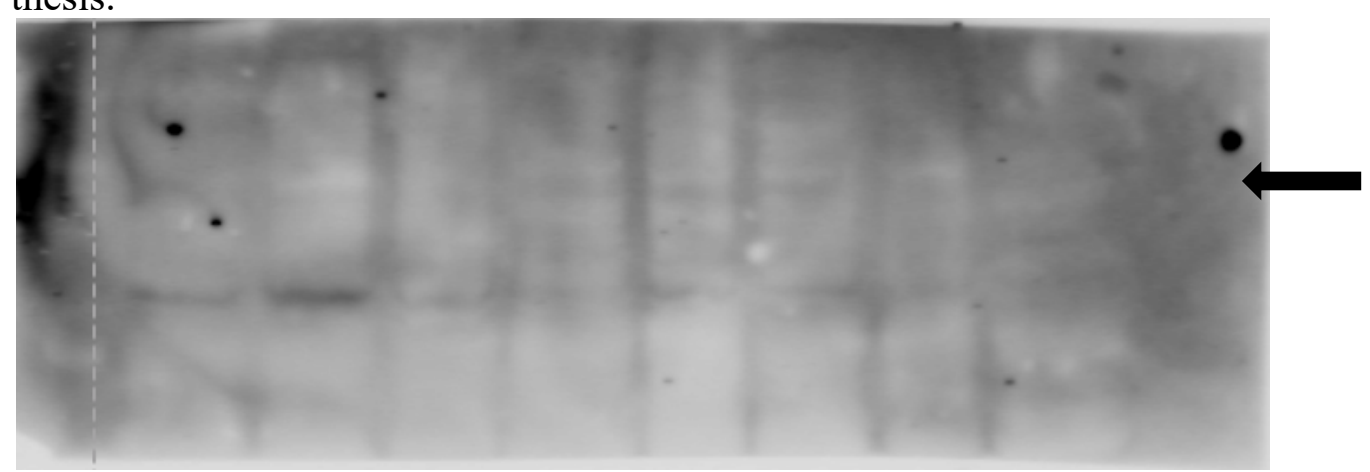

Figure A-1 First attempt at Western blot. Failing to see any protein-phosphorylation around 70 kDalton (arrow), the expected molecular weight of phosphorylated S6K.

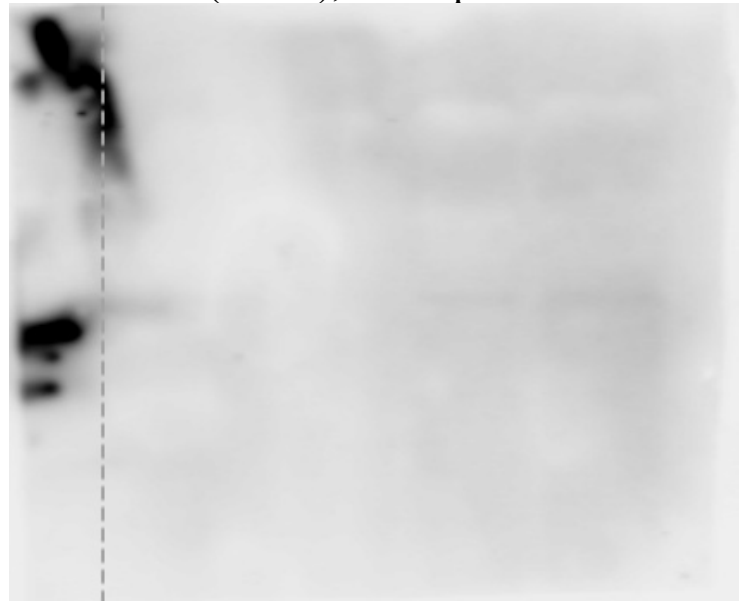

Figure A-2 Second attempt at Western blot for S6K. Again, no visible sign of phosphorylated S6K. 


\section{B Copyright documentation}

Copy right information for Figure 1.1

SPRINGER NATURE LICENSE

TERMS AND CONDITIONS

Oct 23, 2021

This Agreement between Michigan Technological University -- Tessa Steenwinkel ("You") and Springer Nature ("Springer Nature") consists of your license details and the terms and conditions provided by Springer Nature and Copyright Clearance Center.

License Number $\quad 5173750006120$

License date

Oct 21, 2021

Licensed Content Publisher

Springer Nature

Licensed Content Publication

Oncogene

Licensed Content Title

The evolution of the TOR pathway and its role in cancer

Licensed Content Author

E M Beauchamp et al

Licensed Content Date

Dec 17, 2012

Type of Use

Thesis/Dissertation

Requestor type

academic/university or research institute

Format

electronic

Portion

figures/tables/illustrations

Number of

figures/tables/illustrations

1

High-res required no

Will you be translating? no

Circulation/distribution $\quad 1-29$

Author of this Springer Nature no

content

The Effect of Nutrition on Longevity and Fertility in Diverse

Title Drosophila: A TOR-Mediated Process

Institution name

Michigan Technological University

Expected presentation date

Nov 2021

Portions

Figure 2

Requestor Location

Michigan Technological University 